\title{
Free jejunal autograft combined with extensive esophagogastrectomy for unshuntable extrahepatic portal hypertension
}

This is the first report of the use of a free jejunal autograft vascularized by the internal thoracic (internal mammary) artery and vein to restore continuity of the digestive tract after total gastrectomy and distal $65 \%$ esophagectomy for recurrent bleeding esophagogastric varices caused by unshuntable extrahepatic portal hypertension. The procedure was used in two young adults who, because of numerous previous abdominal operations, had a severely scarred and contracted intestinal mesentery that precluded conventional use of the small or large intestine with an intact blood supply to bridge the gap between the upper thoracic esophagus and the abdominal jejunum. Before referral, the two patients had 21 and eight bouts of variceal hemorrhage, respectively, that necessitated a cumulative total of 108 and 74 units of blood transfusion, necessitated 17 and 12 admissions to the hospital, and failed to respond to four and five operations and 14 and 18 sessions of endoscopic sclerotherapy. After extensive esophagogastrectomy combined with a free jejunal autograft, both patients have done well during follow-up of 9 and 3 years, respectively. Both have been in good to excellent health with stable weight, freedom from digestive tract bleeding, normal liver function, and no encephalopathy. These results confirm our recently reported conclusions regarding the uniform long-term effectiveness of extensive esophagogastrectomy in the treatment of unshuntable extrahepatic portal hypertension and suggest that thoracic and general surgeons familiar with microvascular techniques may find the free jejunal autograft to be useful in various circumstances in which it is necessary to replace all or a substantial part of the thoracic esophagus. (J THORAC CardiovaSC SURG 1994;108:346-53)

Marshall J. Orloff, MD, Pat O. Daily, MD, Lisa A. Orloff, MD, San Diego, Calif., and Mark S. Orloff, MD, Rochester, N.Y.

$\mathrm{E}$ occlusion of all major tributaries of the portal venous system is one of the most difficult problems in the field of portal hypertension. Shunts cannot be used in patients with this condition because the portal, superior mesenteric, and splenic veins are not available for construction of a portal-systemic shunt. All of these patients have large esophageal and gastric varices that bleed repeatedly. The repeated bouts of life-threatening hemorrhage are responsible for enormous morbidity, a substantial

From the Department of Surgery, University of California, San Diego, Medical Center, San Diego, Calif.

Received for publication Nov. 8, 1993.

Accepted for publication Feb, 4, 1994.

Address for reprints: Marshall J. Orloff, MD, UCSD Medical Center, 200 West Arbor Dr., San Diego, CA 92103-8999.

Copyright 1994 by Mosby-Year Book, Inc.

$0022-5223 / 94 \$ 3.00+0 \quad 12 / 1 / 55552$ mortality rate, a high risk of blood transfusion-transmitted infection, and large economic costs resulting from numerous hospitalizations and days lost from work. ${ }^{1-13}$

Unshuntable EHPH has been unresponsive to the usual nonshunting measures of treatment. The procedures that have been tried extensively without success include splenectomy, transesophageal ligation of varices, gastroesophageal transections and devascularizations, subtotal and total gastrectomy, distal esophagogastrectomy, and endoscopic sclerotherapy. The record of failure of all conventional forms of treatment led us to adopt a radical therapeutic approach in these patients consisting of one-stage total gastrectomy and $65 \%$ to $75 \%$ distal esophagectomy. Recently, we reported our long-term results in a series of patients who underwent this extensive operation. ${ }^{14}$ Survival was $100 \%$, no patient had recurrent hemorrhage, and the quality of life was good during a follow-up of 1 to 26 years. 
In most of our patients, continuity of the digestive tract has been restored by Roux-en-Y esophagojejunostomy. However, in some patients, because of extensive scarring and contraction of the bowel mesentery that resulted from numerous previous operations, it has not been possible to bring either the small or large intestine with its blood supply intact up into the thorax for anastomosis to the upper third of the esophagus. Under such circumstances, we have successfully bridged the gap between the upper esophagus and abdominal intestine with a long free autograft of jejunum vascularized by microvascular anastomoses to the internal thoracic (internal mammary) artery and vein.

A free jejunal autograft, consisting of a short segment of jejunum anastomosed to blood vessels in the neck by microvascular techniques, has been used extensively by otolaryngologists and plastic surgeons to replace the pharyngoesophagus after resections for cancer of the cervical esophagus and pharynx. During the past 15 years, more than 600 cases of free jejunal autograft in the neck have been reported, with a high rate of success. ${ }^{15-34}$ However, free intestinal autografts have rarely been used to replace the thoracic esophagus, ${ }^{16,21,35-37}$ and no reports have been published of their use in patients with the special problems resulting from EHPH and bleeding varices. The literature suggests that thoracic surgeons and general surgeons are not familiar with the use of free intestinal autografts for replacement of the esophagus. It is the purpose of this communication to describe the technique of placing a long free jejunal autograft in the thorax after extensive esophagogastrectomy and to report two cases in which this technique was used successfully in the treatment of patients with bleeding esophagogastric varices caused by unshuntable EHPH.

\section{Methods}

The operation is performed in one stage through separate midline upper abdominal and median sternotomy incisions. The patient is positioned so that the two incisions can be made without having to change the patient's position, prepare the skin more than once, or redrape the patient. The abdominal incision is made first and the intraabdominal structures are identified by dissecting the extensive dense and vascular adhesions that have resulted from previous operations. A total gastrectomy is performed and the duodenal stump is closed.

A 40 to $50 \mathrm{~cm}$ long segment of jejunum to be used as the free graft is selected as close to the ligament of Treitz as possible. Because of the dense adhesions and scarring of the mesentery, it may be necessary to select a jejunal segment that is located at some distance from the ligament of Treitz. The jejunal segment is divided at each end between noncrushing clamps. The vascular pedicle is then carefully dissected down to its origin from the superior mesenteric artery and vein so as to obtain blood vessels of the greatest possible length and diameter for the subsequent anastomoses in the thorax. The vascular pedicle is not transected until the recipient bed in the thorax has been prepared so that the jejunal segment remains vascularized until the last possible moment.

The free end of the jejunum proximal to the future autograft is anastomosed to the side of the distal jejunum so as to create a 30 to $40 \mathrm{~cm}$ Roux-en- $Y$ limb of jejunum in the abdomen. The anastomosis is done in two layers. The free end of the jejunum distal to the future autograft is left clamped for future anastomosis to the distal end of the autograft.

A median sternotomy is made and the distal two thirds of the esophagus is resected. Either the right or the left internal thoracic artery and vein are dissected free from the undersurface of the sternum and costal cartilages for a considerable length so that they will reach to the vascular pedicle of the future free jejunal autograft.

Attention is then directed to the future free jejunal autograft. Its vascular pedicle is transected and it is gently passed up into the thorax as a free isoperistaltic segment so that its proximal end lies adjacent to the previously transected upper esophagus and its distal end lies adjacent to the previously prepared Rouxen-Y jejunal limb in the abdomen. The patient is heparinized systemically. The internal thoracic artery and vein are transected at a point that will permit their being swung over to the vascular pedicle of the free graft without tension or excessive tortuosity. Microvascular anastomoses are then performed between the internal thoracic vessels and the vessels in the mesenteric pedicle of the free jejunal autograft with interrupted sutures of 7-0 vascular material. The venous anastomosis is performed first and then the arterial anastomosis. Ischemic time is less than 30 minutes. After the FJA has been vascularized, it is trimmed if its length is excessive and secured to the prevertebral fascia with tacking sutures to prevent twisting or pulling on the delicate vascular anastomoses. It is then anastomosed in two layers to the esophagus above and the Roux-en-Y limb of jejunum below. Before completion of these anastomoses a nasogastric tube is threaded through the free graft down into the distal jejunum. The anastomoses are tested for leaks by installation of saline solution through the nasogastric tube. The completed free jejunal autograft is depicted in Fig. 1.

Because of the short ischemic time, we do not perfuse the graft through the jejunal artery with heparin or any of the organ preservation solutions. Moreover, we do not believe it is necessary to subject the graft to hypothermia. Before the abdomen is closed, a temporary feeding jejunostomy is placed just distal to the Roux-en-Y jejunojejunostomy.

Postoperatively, the patients are maintained on nasointestinal suction for 7 to 10 days, after which an esophagogram with the patient swallowing water-soluble contrast material is obtained. If no leak is demonstrated, the nasointestinal tube is removed and oral feedings are started and rapidly advanced from liquids to solids.

\section{Case reports}

CASE 1. A 19-year-old woman was referred to us after her twenty-first bout of bleeding esophagogastric varices with a diagnosis, based on splanchnic angiography, of unshuntable EHPH. She had had four unsuccessful operations that included splenectomy at $1 \frac{1}{2}$ years of age, mesocaval shunt with a synthetic $\mathrm{H}$-graft at $11 \frac{11 / 2}{2}$ years of age, transesophageal ligation of varices at 16 years of age, and gastroesophageal transection and devascularization at 18 years of age. In addition, three series of 


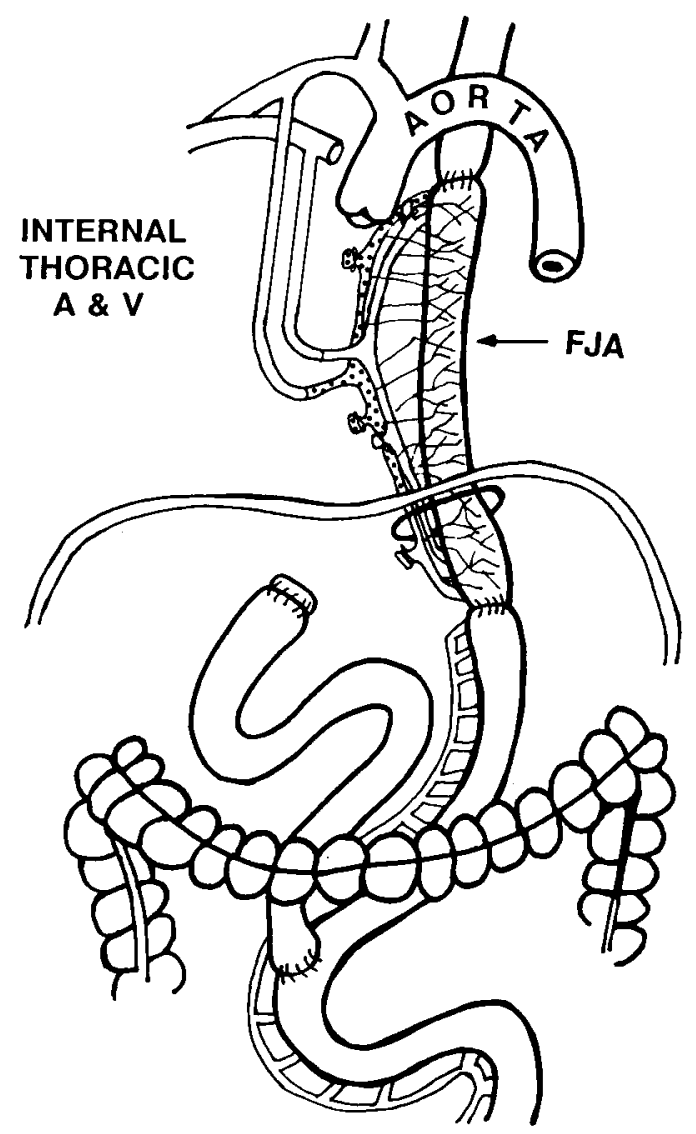

Fig. 1. Total gastrectomy and distal two-thirds esophagectomy with reconstruction by a long free jejunal autograft $(F J A)$ vascularized by the internal thoracic artery and vein $(A \& V)$.

endoscopic sclerotherapy injections (total of 14 sessions) failed to control the variceal bleeding.

One-stage total gastrectomy and resection of the distal two thirds of the esophagus were performed through midline abdominal and median sternotomy incisions. The abdominal portion of the operation, which was performed first, revealed dense vascular adhesions and a severely contracted bowel mesentery from the four previous operations. It was not possible to free up a sufficient length of jejunum to reach the upper part of the thorax for an esophagojejunostomy. Accordingly, a $50 \mathrm{~cm}$ free jejunal autograft was inserted between the esophagus above and a $30 \mathrm{~cm}$ intraabdominal Roux-en-Y limb of jejunum in the abdomen below. The graft was vascularized by the right internal thoracic artery and vein. Results of an operative liver biopsy were reported as within normal limits. The postoperative course was uneventful and the patient was discharged to her home on a diet of solid food on postoperative day 25 . She has had no further bleeding during 9 years of follow-up and has a life of good quality.

CASE 2. A 36-year-old man was referred to us after his eighth episode of variceal hemorrhage with a diagnosis, based on splanchnic angiography, of unshuntable EHPH. He had had five unsuccessful operations that included a mesocaval shunt with a synthetic $\mathrm{H}$-graft 5 years before admission, splenectomy and central splenorenal shunt with a synthetic graft 4 years before admission, a second mesocaval shunt with a synthetic $\mathrm{H}$-graft 1 year before admission, and two exploratory laparotomies $4 \frac{1}{2}$ years and 9 months before admission. During the last operation, portal decompression was found to be impossible. In addition, four series of endoscopic sclerotherapy injections ( total of 18 sessions) were followed by recurrent variceal bleeding. Results of operative liver biopsies were within normal limits.

One-stage total gastrectomy and resection of the distal two thirds of the esophagus were performed through midline abdominal and right posterolateral thoracotomy incisions. The operation was arduous and time-consuming because of large portal-systemic collateral veins, dense vascular intraabdominal adhesions, and a severely contracted bowel mesentery from the five previous operations. Continuity of the digestive tract was restored by a Roux-en-Y esophagojejunostomy, but considerable difficulty was encountered in getting the Roux-en-Y limb of jejunum to reach the upper esophagus. On the tenth postoperative day, an esophagogram demonstrated leakage from the esophagojejunostomy. Accordingly, the intrathoracic jejunal limb was resected, the upper esophagus and abdominal jejunum were stapled shut, and a distal feeding jejunostomy was performed. Seven months later, digestive tract continuity was restored by a $40 \mathrm{~cm}$ long free jejunal autograft between the thoracic esophagus and what remained of the Roux-en-Y jejunal limb in the abdomen. The free jejunal autograft was inserted through midline abdominal and median sternotomy incisions and was vascularized by the right internal thoracic artery and vein. Only $15 \mathrm{~cm}$ remained of the Roux-en-Y limb in the abdomen, but it was not possible to obtain greater length because the jejunum was encased in dense adhesions. The man has had no further bleeding during 3 years of follow-up and his life is of good quality. He has had occasional vomiting from reflux of jejunal contents when he has eaten too rapidly, probably because of the unavoidably short Roux-en-Y limb in the abdomen.

\section{Results}

Clinical data and results of treatment of the two patients who underwent a free jejunal autograft procedure combined with extensive esophagogastrectomy are summarized in Table I. Both of the patients were young adults whose variceal bleeding developed early in life, in infancy in one of them. Visceral angiography at our institution confirmed the previous finding of occlusion of all major tributaries of the portal venous system. Hepatic vein catheterization showed a normal corrected wedged hepatic vein pressure, a finding that is consistent with extrahepatic portal vein thrombosis. Very large esophageal and gastric varices were demonstrated in both patients by endoscopy, barium contrast roentgenography, and visceral angiography. Liver function test results and operative liver biopsy results were within normal limits in both patients, indicating the absence of liver disease.

Both patients had a history of severe bleeding from esophagogastric varices. One patient had had 21 bouts of variceal hemorrhage and the other had had eight episodes of bleeding. The variceal bleeding episodes necessitated a 
Table I. Clinical data on patients treated by free jejunal graft combined with extensive esophagogastrectomy for unshuntable EHPH

\begin{tabular}{|c|c|c|}
\hline & Case 1 & Case 2* \\
\hline Age $(\mathrm{yr})$ at esophagogastrectomy & 19 & 36 \\
\hline Age $(\mathrm{yr})$ at first variceal bleeding & $11 / 2$ & 29 \\
\hline Etiology of EHPH & Omphalitis & Unknown \\
\hline Unshuntable EHPH by angiography & Yes & Yes \\
\hline Corrected wedged hepatic venous pressure & Normal & Normal \\
\hline Esophageal and gastric varices & $4+/ 4+$ & $4+/ 4+$ \\
\hline Liver function tests & Normal & Normal \\
\hline Liver biopsy & Normal & Normal \\
\hline \multicolumn{3}{|l|}{ No. of previous: } \\
\hline Variceal bleeding episodes & 21 & 8 \\
\hline Units of transfused blood & 108 & 74 \\
\hline Hospital admissions & 17 & 12 \\
\hline Unsuccessful operations & 4 & 5 \\
\hline Unsuccessful endoscopic sclerotherapy sessions & 14 & 18 \\
\hline \multicolumn{3}{|l|}{ Operative and hospital data* } \\
\hline \multirow[t]{2}{*}{ Operating time $(\mathrm{hr})$} & 7.0 & I: 17.0 \\
\hline & & II: 7.0 \\
\hline \multirow[t]{2}{*}{ Units of transfused blood } & 7 & I: 24 \\
\hline & & II: 7 \\
\hline \multirow[t]{2}{*}{ Postop. complications } & None & I: Anastomotic leak \\
\hline & & II: None \\
\hline \multirow[t]{2}{*}{ Days in hospital } & 25 & I: 30 \\
\hline & & II: 20 \\
\hline \multicolumn{3}{|l|}{ Follow-up } \\
\hline Y'ears & 9 & 3 \\
\hline Recurrent variceal bleeding & 0 & 0 \\
\hline Portal-systemic encephalopathy at any time & 0 & 0 \\
\hline Endoscopy of free jejunal graft & Normal & Normal \\
\hline General health & Excellent & Good \\
\hline Gainfully employed & Full-time & Intermittent \\
\hline
\end{tabular}

*In case 2, I is the first operation and II is the free jejunal autograft operation.

total of 108 units of transfused blood in one patient and 74 units in the other. The recurrent bouts of variceal bleeding necessitated 17 costly hospital admissions in 17 years in one patient and 12 hospital admissions in 5 years in the other.

Shunting would have been possible in both patients at one time but became impossible as a result of unsuccessful operations. Both patients underwent repeated endoscopic sclerotherapy, but esophageal varices invariably recurred and rebled. Moreover, bleeding developed from large gastric varices that were not amenable to endoscopic sclerotherapy.

Both patients had dense adhesions and severe scarring and contraction of the bowel mesentery that precluded mobilization of a sufficient length of jejunum with its blood supply intact for construction of a tension-free, well-vascularized anastomosis to the proximal esophagus high in the thorax. In fact, an attempt to perform such an anastomosis in the second patient failed and led to the use of a free jejunal autograft as a salvage procedure.
Regular follow-up has been conducted for 9 years and 3 years, respectively, after extensive esophagogastrectomy and replacement of the esophagus with a free jejunal autograft. Neither patient has had any digestive tract bleeding. As we have invariably observed in patients with EHPH, portal-systemic encephalopathy has not occurred in either patient. Endoscopy and barium contrast roentgenograms of the free jejunal autograft at almost yearly intervals have shown a normal-appearing mucosa and rapid emptying of the jejunum. Fig. 2 shows a contrast esophagogram performed 1 year after insertion of the free jejunal autograft in the second patient. Both patients have been in good to excellent health with stable weight, although they weigh less than before the operation. The first patient has been gainfully employed full-time, and the second patient has had intermittent employment.

\section{Discussion}

In 1959 , Seidenberg and associates ${ }^{38}$ reported the first use of a free jejunal autograft to reconstruct the cervical 

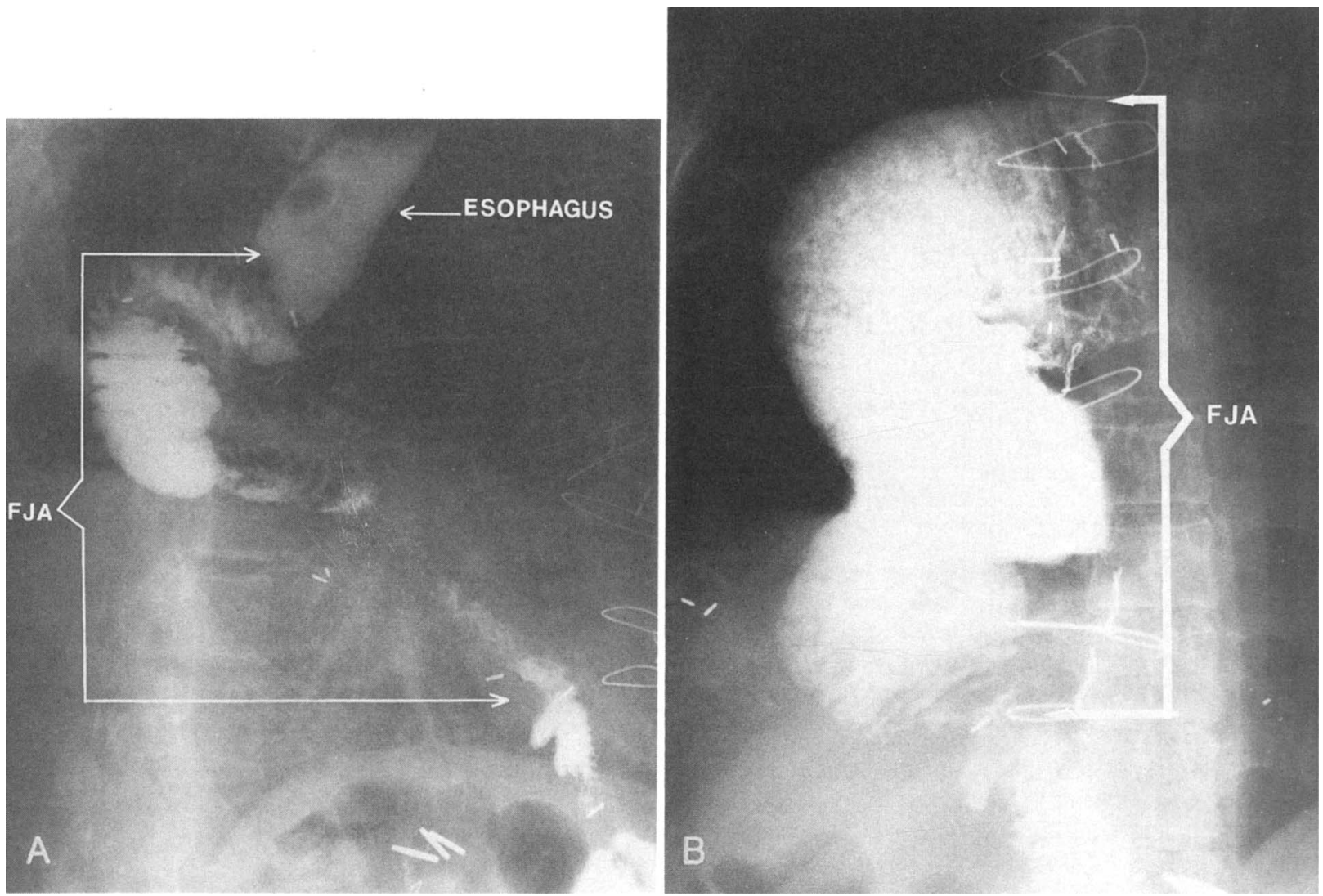

Fig. 2. Barium contrast roentgenograms of the free jejunal autograft $(F J A)$ anastomosed to the upper esophagus and intraabdominal jejunum performed 1 year after insertion of the autograft in case 2.

esophagus after pharyngoesophagectomy for recurrent squamous cell carcinoma. Soon after that, with the introduction of the operating microscope ${ }^{39}$ and the development of precise microvascular techniques, replacement of the cervical esophagus by a short free jejunal autograft anastomosed to blood vessels in the neck became a useful and widely accepted method of treating patients with cancer of the pharyngoesophagus. By 1991 close to 700 cases had been reported in which a free jejunal autograft was used to replace the pharyngoesophagus, with an overall graft viability rate of approximately $90 \%$ and effective swallowing function in approximately $80 \%$ of the patients. ${ }^{15-34}$

In contrast to their frequent use in the neck, free intestinal autografts have rarely been used to replace the thoracic esophagus. Instead, after resection of all or part of the thoracic esophagus, continuity of the digestive tract generally has been restored by gastric pull-up, Roux-en-Y esophagojejunostomy, or interposition of jejunum or colon with its vascular pedicle intact. However, these conventional methods of reconstruction are not possible when a total gastrectomy has been done or when the small or large intestine will not reach to the upper thorax or neck because of scarring and contraction of the bowel mesentery. Under such circumstances, use of a free jejunal autograft may be the only means of restoring digestive tract continuity.

The free jejunal autograft has been used successfully to replace the upper thoracic esophagus in a handful of patients with benign corrosive strictures. ${ }^{16,35,36} \mathrm{In}$ all of these cases, the free jejunal autograft was short and the mesenteric blood vessels were anastomosed to blood vessels in the neck, much as in the grafts used to replace the pharyngoesophagus. Only two cases have been reported in which a long free jejunal autograft was used to replace the thoracic esophagus and was vascularized by anastomosis to other than cervical blood vessels. Berger and coworkers $^{21}$ successfully used a $27 \mathrm{~cm}$ free jejunal autograft vascularized by the internal thoracic artery and azygos vein to replace the entire thoracic esophagus in an 11-year-old child with a severe corrosive stricture. The free jejunal autograft was anastomosed to the pharynx 
above and the stomach below. McDonald, Rohr, and Tucker ${ }^{37}$ performed a total thoracic esophagectomy for a lye stricture in a 22-year-old man and successfully replaced the esophagus with a free jejunal autograft vascularized above by the innominate artery and vein and below by the left gastric artery and vein. Tension on the inferior vascular anastomoses required interposition of saphenous vein grafts.

The two cases described in the present report represent the first use of a free jejunal autograft for replacement of a substantial segment of thoracic esophagus in the treatment of portal hypertension and bleeding esophagogastric varices. Moreover, these are the first two cases in which the internal thoracic artery and vein were used to vascularize a free intestinal graft. In both of these cases, tension-free vascular anastomoses were accomplished without difficulty, and the long-term results were satisfactory.

Several technical aspects of the operation warrant emphasis. A conventional purging and antibiotic bowel preparation have been used before the operation in all of our patients undergoing radical esophagogastrectomy because of the outside possibility that it might be necessary to use the colon to replace the esophagus. The patients are positioned on the operating table so as to make it possible to perform both the abdominal and thoracic incisions in continuity. The abdominal part of the operation is performed initially because the findings in the abdomen determine which type of thoracotomy incision will be used. We have used a median sternotomy incision only if the intestinal mesentery is so scarred and contracted that a free jejunal autograft will be required. Because the ischemic time of the free jejunal autograft should regularly be less than 60 minutes and usually less than 30 minutes, we do not believe it is necessary to perfuse the autograft with an organ preservation solution or to cool the graft below room temperature. However, we have used systemic heparinization beginning just before transplantation of the free jejunal autograft to prevent clotting of the vascular anastomoses. To minimize the duration of ischemia, we do not transect the vascular pedicle of the segment of jejunum to be used as a free autograft until the recipient bed in the thorax is ready to receive the free graft and the internal thoracic artery and vein have been prepared for anastomosis. The jejunum is inserted in an isoperistaltic direction in the hope that so doing will facilitate swallowing. Considerable difference of opinion has been expressed about the best sequence for performing the vascular and enteric anastomoses. ${ }^{15,22-25,27-30,31,33,36} \mathrm{We}$ advocate performing the vascular anastomoses initially, venous then arterial, to minimize the duration of graft ischemia. Then we perform the upper anastomosis of free jejunum to esophagus in the thorax, and finally we anastomose the free jejunum to the abdominal jejunum. To avoid twisting or disruption of the vascular anastomoses, we fix the free jejunal autograft to the prevertebral fascia before undertaking the enteric anastomoses.

The quality of motor function of the free jejunal autograft is uncertain. Dysphagia is common in patients with a free jejunal autograft in the neck, but some or most of this disturbance may be due to interference with the swallowing mechanism caused by pharyngoesophagectomy. Radiographic, manometric, and electrical studies in animals ${ }^{40}$ and human beings ${ }^{41,42}$ with a free jejunal autograft have yielded conflicting results, but the weight of evidence indicates that the free jejunal autograft acts as a passive conduit of food. The denervated autograft in the neck shows fasting motor activity that is not synchronous with that of the intact abdominal jejunum, and it does not propel a food bolus in a coordinated fashion or respond to feeding normally.

Patients with unshuntable EHPH have received a wide variety of therapeutic measures in a concerted and often desperate attempt to control their variceal bleeding, without success. Because of the consistent record of failure of conventional therapeutic measures, some years ago we undertook a radical therapeutic approach to unshuntable EHPH consisting of one-stage total gastrectomy and $65 \%$ to $75 \%$ distal esophagectomy. No operative deaths occurred in a series of 18 patients, all patients are currently alive, and none had recurrent bleeding during 1 to 26 years of follow-up (mean 13.9 years, 7 or more years in 14 patients). ${ }^{14}$ Quality of life was good. Portal-systemic encephalopathy did not develop, liver function remained normal, general health remained excellent or good, and $88 \%$ of the patients resumed gainful employment or full-time housekeeping. Most of the operations were long and arduous because of the scarring and adhesions from previous abdominal procedures. However, in most patients it was possible to reconstruct the digestive tract by a Roux-en-Y esophagojejunostomy using a long limb of jejunum with an intact mesenteric blood supply on a pedicle. In the two patients described in the present report, the intestinal mesentery was so scarred and contracted that it was not possible to fashion a viable anastomosis between the upper esophagus and any segment of the small or large intestine with its mesenteric blood supply intact. Under such circumstances, the only reasonable alternative was the use of a free intestinal autograft to bridge the gap between the abdominal jejunum and the upper esophagus. Use of a free jejunal autograft vascularized by the internal thoracic artery and vein proved to be a satisfactory solution to this difficult problem. The favorable long-term results obtained with an intrathorac- 
ic free jejunal autograft suggest that this procedure may be useful in a variety of circumstances in which it is necessary to replace all or much of the thoracic esophagus.

\section{R EFERENCES}

1. Shumacker HB, King H. Splenic studies. II. Portal hypertension in children associated with gastroesophageal hemorrhage. Arch Surg 1952;65:499-510.

2. King H, Shumacker HB Jr. Venous shunt procedures for portal hypertension in children. Surgery 1958;43:680-2.

3. Habif DV. Treatment of esophageal varices by partial esophagogastrectomy and interposed jejunal segment. Surgery $1959 ; 46: 212-35$.

4. Hallenbeck GA, Adson MA. Esophagogastric varices without hepatic cirrhosis. Arch Surg 1961;83:370-83.

5. Voorhees AB Jr, Harris RC, Britton RC, Price JB, Santulli TV. Portal hypertension in children: 98 cases. Surgery 1965;58:540-9.

6. Voorhees AB Jr, Price JB Jr. Extrahepatic portal hypertension: a retrospective analysis of 129 cases and associated clinical implications. Arch Surg 1974;108:338-41.

7. Bernstein EF, Varco RL, Wangensteen OH. Treatment of bleeding esophageal varices in portal-systemic shunt failures. Arch Surg 1969;99:171-8.

8. Hamilton DW, Hunt AH. Extrahepatic portal obstruction. Med J Aust 1970;1:493-9.

9. Rothwell-Jackson RL, Hunt AH. Proximal gastric resection in the treatment of bleeding gastro-oesophageal varices in patients with portal hypertension due to extrahepatic obstruction. Br J Surg 1970;57:487-94.

10. Fonkalsrud EW, Myers NA, Robinson MJ. Management of extrahepatic portal hypertension in children. Ann Surg 1974;180:487-93.

11. Fonkalsrud EW. Surgical management of portal hypertension in children. Arch Surg 1980;1 15:1042-5.

12. Macpherson AIS. Portal hypertension in children: an experience of 33 cases. Scott Med J 1984;29:6-14.

13. Warren WD, Henderson JM, Millikan WJ, Galambos JT, Bryan FC. Management of variceal bleeding in patients with noncirrhotic portal vein thrombosis. Ann Surg 1988; 207:623-34.

14. Orloff MJ, Orloff MS, Daily PO, Girard B. Long-term results of radical esophagogastrectomy for bleeding varices due to unshuntable extrahepatic portal hypertension. Am J Surg 1993;167:96-103.

15. Shangold LM, Urken ML, Lawson W. Jejunal transplantation for pharyngoesophageal reconstruction. Otolaryngol Clin North Am 1991;24:1321-42.

16. Nakamura T, Inokuchi K, Sugimachi K. Use of revascularized jejunum as a free graft for cervical esophagus. Jpn J Surg 1975;5:92-102.

17. McKee DM, Peters CM. Reconstruction of the hypopharynx and cervical esophagus with microvascular jejunal

- transplant. Clin Plast Surg 1978;5:305-12.
18. Robinson DW, MacLeod A. Microvascular free jejunum transfer. Br J Plast Surg 1982;35:258-67.

19. Kato H, Watanabe H, Iizuka T, et al. Primary esophageal reconstruction after resection of the cancer in the hypopharynx or cervical esophagus: comparison of free forearm skin tube flap, free jejunal transplantation and pull-through esophagectomy. Jpn J Clin Oncol 1987;17: 255-61.

20. Reuther JF, Steinau H, Wagner R. Reconstruction of large defects in the oropharynx with a revascularized intestinal graft: an experimental and clinical report. Plast Reconstr Surg 1984;73:345-58.

21. Berger A, Tizian C, Hausamen J, Schulz-Coulon H, Löhlein D. Free jejunal graft for reconstruction of oral, oropharyngeal and pharyngoesophageal defects. J Reconstr Microsurg 1984;1:83-94.

22. Chang T, Wang W, Huang O. One-stage reconstruction of esophageal defect by free transfer of jejunum: treatment and complications. Ann Plast Surg 1985;54:492-6.

23. Nozaki M, Huang TT, Hayashi M, Endo M, Hirayama T. Reconstruction of the pharyngoesophagus following pharyngoesophagectomy and irradiation therapy. Plast Reconstr Surg 1985;76:386-94.

24. Wang ID, Sun YE, Chen Y. Free jejunal grafts for reconstruction of the pharynx and cervical esophagus. Ann Otol Rhinol Laryngol 1986;95:348-51.

25. Theile DE, Robinson DW, McCafferty GJ. Pharyngolaryngectomy reconstruction by revascularized free jejunal graft. Aust N Z J Surg 1986;56:849-52.

26. Biel MA, Maisel RH. Free jejunal autograft reconstruction of the pharyngoesophagus: review of a 10-year experience. Otolaryngol Head Neck Surg 1987;96:369-75.

27. Deane LM, Gilbert DA, Schecter GL, Baker JW. Free jejunal transfer for the reconstruction of pharyngeal and cervical esophageal defects. Ann Plast Surg 1987;19:499503.

28. Salamoun W, Swartz WM, Johnson JT, et al. Free jejunal transfer for reconstruction of the laryngopharynx. Otolaryngol Head Neck Surg 1987;96:149-50:

29. Ferguson JL, DeSanto LW. Total pharyngolaryngectomy and cervical esophagectomy with jejunal autotransplant reconstruction: complications and results. Laryngoscope 1988;98:911-4.

30. Shumrick DA, Savoury LW. Recent advances in laryngo, pharyngo, esophageal reconstruction. Acta Otolaryngol (Stockh) 1988;458(Suppl):190-3.

31. Flynn MB, Banis J, Acland R. Reconstruction with free bowel autografts after pharyngoesophageal or laryngopharyngoesophageal resection. Am J Surg 1989; 158:333-6.

32. Coleman JJ, Tan K, Searles JM, Hester TR, Nahai F. Jejunal free autograft: analysis of complications and their resolution. Plast Reconstr Surg 1989;84:589-98.

33. Fisher SR, Cameron R, Hoyt DJ, Cole TB, Seigler HF, Meyers WC. Free jejunal interposition graft for recon- 
struction of the esophagus. Head Neck Surg 1990;12:12630 .

34. Schusterman MA, Shestak K, deVries EJ, et al. Reconstruction of the cervical esophagus: free jejunal transfer versus gastric pull-up. Plast Reconstr Surg 1990;85:16-21.

35. Prevot J, Lepelley M, Schmitt M. Small bowel esophagoplasty with vascular microanastomoses in the neck for treatment of esophageal burns in childhood. Prog Pediatr Surg 1985;18:108-17.

36. Jones BM, Gustavson EH. Free jejunal transfer for reconstruction of the cervical oesophagus in children: a report of two cases. Br J Plast Surg 1983;36:162-7.

37. McDonald JC, Rohr MS, Tucker WY. Recent experiences with autotransplantation of the kidney, jejunum and pancreas. Ann Surg 1983;197:678-87.
38. Seidenberg B, Rosznak SS, Hurwitt ES, Som ML. Immediate reconstruction of the cervical esophagus by a revascularized isolated jejunal segment. Ann Surg 1959;149: $162-71$.

39. Jacobson $\mathbf{J H}$, Suarez EL. Microsurgery in anastomosis of small vessels. Surg Forum 1960;11:243-5.

40. Scher N, Wiederhold ML, Garza JR, Pingree TF, Haughey $\mathrm{BH}$. Baseline motility of the free jejunal graft in a new canine model. Am J Otolaryngol 1990;11:407-15.

41. Kerlin P, McCafferty GJ, Robinson DW, Theile D. Function of a free jejunal "conduit" graft in the cervical esophagus. Gastroenterology 1986;90:1956-63.

42. Meyers WC, Seigler HF, Hanks JB, et al. Postoperative function of free jejunal transplants for replacement of the cervical esophagus. Ann Surg 1980;192:439-50.

\section{Bound volumes available to subscribers}

Bound volumes of THE JOURNAL OF THORACIC AND CARDIOVASCULAR SURGERY are available to subscribers (oniy) for the 1994 issues from the Publisher, at a cost of $\$ 82.50$ for domestic, $\$ 106.28$ for Canadian, and $\$ 100.50$ for international subscribers for Vol. 107 (January-June) and Vol. 108 (July-December). Shipping charges are included. Each bound volume contains a subject and author index and all advertising is removed. Copies are shipped within 60 days after publication of the last issue of the volume. The binding is durable buckram with the JOURNAL name, volume number, and year stamped in gold on the spine. Payment must accompany all orders. Contact Mosby-Year Book, Inc., Subscription Services, 11830 Westline Industrial Drive, St. Louis, Missouri 63146-3318, USA; phone 1 (800) $453-$ 4351 or (314) 453-4351.

Subscriptions must be in force to qualify. Bound volumes are not available in place of a regular JouRNAL subscription. 